\title{
INNOVATION AND IMPROVEMENT \\ Health Literacy in Transitions of Care: An Innovative Objective Structured Clinical Examination for Fourth-Year Medical Students in an Internship Preparation Course
}

\author{
Kimberly Bloom-Feshbach, $\mathrm{MD}^{7}$, Dana Casey, $\mathrm{MD}^{7}$, Lucy Schulson, MD, MPH ${ }^{7}$, Peter Gliatto, MD ${ }^{7}$, \\ Jonathan Giftos, $\mathrm{MD}^{2}$, and Reena Karani, MD, MHPE ${ }^{7}$ \\ ${ }^{1}$ Icahn School of Medicine at Mount Sinai, New York, NY, USA; ${ }^{2}$ Albert Einstein College of Medicine, Bronx, NY, USA.
}

BACKGROUND: Low health literacy is associated with adverse health outcomes, especially during transitions of care. Competency-based assessments may improve communication during this time.

AIM: To develop an Objective Structured Clinical Examination (OSCE) for medical students to demonstrate communication skills to be used during the hospital discharge process with patients of low health literacy.

SETTING: The OSCE was integrated into the curriculum of an internship preparatory clerkship.

PARTICIPANTS: One hundred and one fourth-year medical students participated.

PROGRAM DESCRIPTION: Students received a skillsbased health literacy workshop. In the OSCE, learners counseled standardized patients regarding initiation of anticoagulation at discharge and wrote discharge instructions.

PROGRAM EVALUATION: Fifty-seven students completed the workshop prior to the OSCE, and 44 participated in the workshop after the completing the OSCE. Participants who completed the workshop first outperformed their peers on the checklist (15.1 vs. $13.4, p<0.0001$ ) and on the reading level of their written instructions (9.9 vs. 10.6, $p=0.01) ; 82 \%$ felt confident communicating with patients of low health literacy after the workshop and OSCE.

DISCUSSION: This OSCE is a tool to train and evaluate future interns' ability to communicate with patients of limited health literacy levels at hospital discharge. Such innovations may make this period of time safer for patients, improving health outcomes.

KEY WORDS: OSCE; medical student; transitions; health literacy.

J Gen Intern Med 31(2):242-6

DOI: $10.1007 / \mathrm{s} 11606-015-3513-1$

(c) Society of General Internal Medicine 2015

\section{INTRODUCTION}

Only $12 \%$ of Americans are considered to have proficient health literacy, and low health literacy is associated with multiple adverse health outcomes, including increased

$\overline{\text { Kimberly Bloom-Feshbach, Dana Casey and Lucy Schulson are joint first }}$ authors.

Received March 17, 2015

Revised July 27, 2015

Accepted August 28, 2015

Published online October 9, 2015 mortality. ${ }^{1-5}$ Patients are at increased risk of adverse events in the period following hospital discharge. ${ }^{6-8}$ Ineffective transitions of care can be a significant source of health disparities, and for patients with low health literacy, communication failures during this time increased the risk of poorer health outcomes and readmissions. ${ }^{9-12}$ Despite the prevalence of low health literacy and the associated risk of significant morbidity and mortality, health professionals are often not trained in communicating with this vulnerable patient population. ${ }^{13}$

Medical school initiatives, including longitudinal curricula on effective communication techniques and Objective Structured Clinical Examinations (OSCEs), have attempted to address this gap in provider training. ${ }^{10,11,14-16}$ The majority of structured health literacy learning opportunities are offered during the first three years of medical school, with fewer than $15 \%$ occurring during the fourth year. ${ }^{13} \mathrm{~A}$ fourth-year student is poised to become an intern. In the inpatient setting, interns shoulder the responsibility of communicating with patients about their diagnoses and ensuring safe hospital discharge. However, published health literacy curricula have not focused on this high-risk period for patients or on the skills needed to ensure that patients with limited health literacy are safely discharged from the hospital.

This evidence-based initiative for fourth-year medical students includes two components: 1) an interactive workshop on screening tools and communication techniques to care for patients with low health literacy, and 2) an innovative OSCE that assesses the application of these skills during transitions of care such as hospital discharge. This OSCE is innovative for several reasons. First, it targets fourth-year medical students as part of their preparation for residency. In addition, it specifically focuses on the discharge process, because effective communication during such a transition is critical for patient safety and is an important skill for all future interns. Finally, it allows learners to apply their knowledge and practice their skills in a safe and simulated yet realistic environment.

\section{SETTING AND PARTICIPANTS}

The workshop and OSCE were integrated into the Introduction-to-Internship Clerkship, a mandatory 
learning experience during the spring semester of the fourth year. One hundred and one fourth-year students participated in the workshop and OSCE between January and April 2014. Fifty-seven students (56\%) completed the workshop, followed by the OSCE 1 week later, while the remaining students $(44 \%)$ completed the OSCE first, providing a natural comparison group in the study, as allocation was based solely upon availability of the OSCE center (Table 1). Gender and specialty choice were recorded for all participants. Twenty-seven students in the fourth-year class were unable to participate because they were in residency interviews or were ill at the time.

\section{PROGRAM DESCRIPTION}

\section{Goals}

The primary goal of this project was to develop an OSCE for fourth-year medical students in order to demonstrate oral and written communication skills to be used during the hospital discharge process with patients of low health literacy.

\section{Educational Methods and Rationale}

The interactive health literacy workshop is modeled on Kripalani and colleagues' workshop for medical residents. ${ }^{15}$ Its objectives are to prepare students to apply knowledge and techniques in the clinical assessment of health literacy level, and to communicate medical information to patients for whom low health literacy is a barrier to care. The OSCE assesses learners' ability to apply the knowledge and demonstrate practical skills from the workshop in the context of hospital discharge involving a patient with low health literacy. ${ }^{9,17}$

\section{Implementation}

We developed an original OSCE scenario, including a clinical case for the students and standardized patients (SPs) (Appendix 1). We piloted the OSCE with a group of four students who had completed all core third-year clerkships

Table 1 Student Characteristics: Students Pursuing Residency Training in Primary Care had Similar OSCE Scores Compared with Classmates Entering Non-Primary Care Specialties $(p=0.5)$

\begin{tabular}{llll}
\hline \hline Characteristic & $\begin{array}{l}\text { Total } \\
(\boldsymbol{n}=\mathbf{1 0 1})\end{array}$ & $\begin{array}{l}\text { Group 1*} \\
(\boldsymbol{n}=\mathbf{5 7})\end{array}$ & $\begin{array}{l}\text { Group 2*} \\
(\boldsymbol{n}=\mathbf{4 4})\end{array}$ \\
\hline $\begin{array}{l}\text { Gender } \\
\text { Male }\end{array}$ & $51(50)$ & $29(51)$ & $22(50)$ \\
$\begin{array}{l}\text { Female } \\
\text { Residency }\end{array}$ & $50(50)$ & $28(49)$ & $22(50)$ \\
$\begin{array}{l}\text { Primary care } \\
\text { Non-primary care }\end{array}$ & $44(44)$ & $27(47)$ & $17(39)$ \\
\hline
\end{tabular}

* Group $1=$ group that completed the workshop prior to OSCE; Group $2=$ control group that completed OSCE prior to workshop

${ }^{\prime}$ Primary care $=$ family medicine, internal medicine, pediatrics and were on scholarly leave before fourth year. Pilot data was used to clarify OSCE instructions for students, ensure that time allotted was reasonable, and improve SP case portrayal. We developed an SP training session in which actors participated in the health literacy skills workshop and watched supplemental videos of patients with low health literacy interacting with the healthcare system. They rehearsed and provided feedback on sample scripts. The SPs were blinded as to which group the students were in. The study was reviewed and deemed exempt by the institutional review board.

Workshop. The student leaders of the health literacy group (KBF, DC, LS) taught the workshop to their peers. Although the workshop is PowerPoint-based, it was designed to be interactive, with many opportunities for student participation and role-play. The workshop emphasizes the importance of health literacy, teaches ways to actively assess health literacy, and allows for the practice of communication skills such as the use of plain language, teach-back, and the method of limiting key information to three major points as outlined in the Ask Me 3 campaign. ${ }^{18,} 19$

OSCE Scenario. The OSCE tasked students with preparing a patient for hospital discharge. Students were instructed to screen the SP for low health literacy and to provide education regarding diagnosis (pulmonary embolus), management (warfarin), and follow-up (international normalized ratio [INR] monitoring). Following completion of the SP encounter, students were directed to write discharge instructions appropriate for the patient. Students received 15 minutes to complete the patient encounter and 15 minutes to complete the written discharge instructions.

Checklist. Our 17-item checklist (Table 2) includes behavioral statements linked to health literacy assessment and discharge planning described in the literature. ${ }^{19-24}$ It assesses performance in health literacy screening, the use of plain language, delivery of a focused message regarding diagnosis and management, and the use of open-ended questions and teach-back, among other evidence-based communication techniques. English proficiency is not evaluated. The trained SPs completed the checklist immediately following each student encounter.

Feedback. Each student viewed his or her scored checklist the day after the OSCE, thus allowing for use of the OSCE not only as a means of assessment but also as a learning tool for participants. After the workshop and OSCE, 65 students $(64 \%)$ completed a voluntary ten-item anonymous survey (Appendix 2) to assess perceptions of the OSCE and workshop effectiveness, the importance of health literacy, self-assessment of effective communication with patients of low health 
Table 2 Checklist for Health Literacy OSCE: This Checklist was Designed to Represent Core Competencies in Health Literacy, and Completed by Trained SPs to Assess Students' Performance. The Mean Score was 14.4/17; Students who Completed the Workshop Prior to the OSCE Outperformed Peers (15.1 vs. 13.4, $p<0.0001)$

1. Asks patient about his/her understanding of diagnosis

2. Asks the following validated health literacy screening question: "How confident are you filling out medical forms yourself?"

3. plains main problem ex., "You had a pulmonary embolus. That means a blood clot in your lung."

4. Explains what patient needs to do ex., "Take warfarin/blood thinner medicine to stop future clots."

5. Explains why treatment is important ex. "Clots are a danger to you. This medicine thins your blood and stops clots."

6. Employs teach-back method at least once ex., "If you were to tell a friend how to take this medication, what would you say?" or "Tell me what foods you need to avoid."

7. Student normalizes teach-back or puts the burden on himself or herself ex., "I do this with all my patients" or "I want to make sure I explained things clearly."

8. Asks at least ONE open-ended question to clarify patient's understanding ex., asks "What questions do you have for me?" instead of yes/no questions such as "Do you understand?" or "Do you have any questions?"

9. Clarifies misunderstanding in nonjudgmental manner ex., "I must not have explained that well. Let me try again..."

10. Repeats key points at least once ex., "As we talked about, you had a clot in your lungs. You must take a blood thinner for at least 3 months."

11. Gives orienting statements ex., "If it's OK, I'd like to talk with you about your new medication."ex., "First I will ask you some questions. Then I will explain what happens when you go home."

12. Uses plain language; avoids medical jargon unless immediately defined/clarifies what is meant ex., may say "pulmonary embolus," but must clarify this as "blood clot in the lung" ex., may say "anticoagulant," but must clarify this as a "blood thinner"

13. Explanations give appropriate amount of information without making the patient feel overwhelmed. When talking with patients of low health literacy, clinicians should deliver focused messages, emphasizing only $1-3$ points per topic ex., discussing only 1-3 side effects of a medication

14. Avoids long sentences (sentences with multiple clauses)

15. Speaks slowly and clearly

16. Speaks at a normal volume

17. Makes patient feel respected. Student is not condescending or judgmental; treats patient as a partner, and elicits patient's perspective

\section{Yes/No \\ Yes/No \\ Yes/No \\ Yes/No \\ Yes/No \\ Yes/No \\ Yes/No \\ Yes/No \\ Yes/No \\ Yes/No}

Most of the time/some of the time/rarely or never

Most of the time/some of the time/rarely or never

Most of the time/some of the time/rarely or never

Most of the time/some of the time/rarely or never

Most of the time/some of the time/rarely or never

Most of the time/some of the time/rarely or never

Most of the time/some of the time/rarely or never literacy, and relevance of the skills taught in the workshop to providing patient care.

\section{PROGRAM EVALUATION}

Checklist. The mean score on the OSCE checklist across the entire group of study participants was 14.4/17 (range, 8.5-17). The Student $t$ test was performed to assess the relationship between workshop participation prior to the OSCE and the final performance score on the OSCE. Chi-square analysis was utilized to assess the relationship between workshop participation prior to the OSCE and each checklist item. Students who completed the workshop before the OSCE outperformed peers, with an average checklist score of 15.1 (range, 10-17), compared to 13.4 (range, 8.5-17) among those who completed the workshop after the OSCE $(p<0.0001)$. This relationship remained statistically significant after controlling for intended career in primary care (defined as family medicine, internal medicine, or pediatrics) versus non-primary care specialty $(p<0.0001)$. The most commonly missed item was question 2: only 33/101 students asked a validated health literacy screening question. However, 51\% (29/57) of students who completed the workshop before the OSCE asked a validated screening question, versus $9 \%(4 / 44)$ of students who completed the workshop after the OSCE $(p<0.0001)$. Students who completed the workshop first were also more likely to use $(p=0.03)$ and normalize teach-back $(p=0.0003)$. Among the entire group, students pursuing residencies in primary care had scores similar to those of their classmates entering non-primary care fields $(p=0.5)$.

Discharge Instructions. We used the Flesch-Kincaid readability score, the SMOG [Simple Measure of Gobbledygook] index, and the Gunning Fog score to assess the reading level of the students' written discharge instructions. The average score among all students across readability tests was a tenth grade reading level (range, grades 7-16) ${ }^{25-28}$ On average, the written discharge instructions of students who completed the workshop before the OSCE were at a grade reading level of 9.9 , versus 10.6 among those who completed it after the OSCE (effect size $0.7, p=0.01$ ).

Survey Results. After completion of the workshop and OSCE, $87 \%$ of students reported confidence (agree or strongly agree) in assessing health literacy, and $82 \%$ felt confident (agree or strongly agree) in effectively communicating with patients of low health literacy. 
Ninety-seven percent of participants agreed that health literacy was important to consider in patient care.

\section{DISCUSSION}

The Health Literacy in Transitions of Care OSCE focuses on imparting communication skills necessary to optimize safety in transitions of care. The OSCE and skill-based workshop were successfully integrated into the existing fourth-year curriculum. The OSCE allowed students to put health literacy theory and communication strategies into practice in a safe yet realistic environment.

Our data indicate that the workshop was effective in improving students' ability to communicate with patients with low health literacy. There was statistically significant higher performance on the checklist among students who completed the workshop prior to the OSCE than among their peers who did not. Workshop participation was most closely associated with improved communication skills in asking validated screening questions and in employing and normalizing teach-back, all clinically important techniques for effective communication with patients with low health literary. With regard to the written task, although there was a statistically significant difference between the two groups, the effect size was less than one reading grade level, and both groups wrote their instructions at a level above which most adults can read, which suggests that we may need to bolster the portion of the curriculum focusing on competency in writing skills for communicating with patients of limited health literacy.

Our educational experience was built on existing curricula in the literature. Kripalani et al. developed an interactive health literacy workshop, filmed SP encounters, and created a feedback opportunity and survey for medical residents. The trainees in this study found the experience meaningful, and noted that it would influence their clinical practice. ${ }^{15}$ Like Roberts et al., we included an emphasis on teach-back as a tool for health literacy communication. ${ }^{11}$ We also used a concept similar to the curricula described by Harper et al., designing our OSCE both as a method of teaching health literacy and as a tool to assess curricular efficacy. ${ }^{29}$ However, our OSCE is innovative in that it targets fourth-year medical students preparing for internship, and unlike existing OSCEs, our encounter focused on the high-risk period of hospital discharge. Our targeting of fourth-year students is especially timely given the growing need for fourth-year assessments that document competencies in entrustable professional activities. $^{30}$

There are several limitations to our study. We were unable to control for variance in educational exposure to health literacy prior to the clerkship. In addition, due to limited resources, we were only able to present students with one OSCE station. In the future, implementing multiple cases would provide more reliable data. Though we attempted to standardize the
OSCE with rigorous SP training, the uniformity with which different SPs assessed students was not measured. Our results would be enhanced by having more than one rater per student encounter and by assessing inter-rater reliability. There was no significant difference in specialty choice or demographics between students who completed the workshop before versus after the OSCE. However, because the groups were not randomized, other differences between the control and intervention groups may have influenced performance. Additionally, there may have been sharing of OSCE materials between the two groups. Finally, we were unable to assess the efficacy of the workshop and OSCE in changing future clinical practice.

Transitions of care are a vulnerable time for all patients, but particularly for those with low health literacy. The Health Literacy in Transitions of Care OSCE is an innovative tool for training future interns and interprofessional learners to identify and better communicate with patients of all health literacy levels during the hospital discharge process. Approximately $50 \%$ of US medical schools with health literacy curricula already use simulated patient encounters, which suggests that our curricular design could be easily applied at other institutions. ${ }^{13}$ Our hope is that widespread health literacy training in medical school will lead to less miscommunication and to improved outcomes for vulnerable populations.

Acknowledgments: A grant from the Arnold P. Gold Foundation provided the funding for our OSCE. We thank the Foundation for its generous support. We are also grateful to the Morchand Center for Clinical Competence at the ISMMS (BF, TS, DF, LA, AF, and all SPS). We thank Dr. Uma Ayyala, and alumni SB, JF, JN, and SS.

Conflict of Interest: The authors declare that they do not have a conflict of interest.

Corresponding Author: Reena Karani, MD, MHPE; Icahn School of Medicine at Mount Sinai, Box 1257, 1 Gustave Levy Place, New York, NY 10029, USA (e-mail: reena.karani@mssm.edu).

\section{REFERENCES}

1. Health Literacy. A Prescription to End Confusion, Committee on Health Literacy. Washington: Institute of Medicine; 2004.

2. Baker DW, Wolf MS, Feinglass J, Thompson JA. Health literacy, cognitive abilities, and mortality among elderly persons. J Gen Intern Med. 2008;23(6):723-6. doi:10.1007/s11606-008-0566-4.

3. Wu J-R, Holmes GM, Dewalt DA, et al. Low literacy is associated with increased risk of hospitalization and death among individuals with heart failure. J Gen Intern Med. 2013;28(9):1174-80. doi:10.1007/s11606-0132394-4

4. Baker DW, Gazmararian JA, Williams MV, et al. Functional health literacy and the risk of hospital admission among Medicare managed care enrollees. Am J Public Health. 2002;92(8): 1278-83.

5. Baker DW, Parker RM, Williams MV, Clark wS. Health literacy and the risk of hospital admission. J Gen Intern Med. 1998;13(12):791-8.

6. Naylor MD, Aiken LH, Kurtzman ET, Olds DM, Hirschman KB. The Importance Of Transitional Care In Achieving Health Reform. Health Aff (Millwood). 2011;30(4):746-54. doi:10.1377/hlthaff.2011.0041.

7. Bell CM, Brener SS, Gunraj N, et al. Association of ICU or hospital admission with unintentional discontinuation of medications for chronic diseases. JAMA. 2011;306(8):840-7. doi:10.1001/jama.2011.1206. 
8. Forster AJ, Murff HJ, Peterson JF, Gandhi TK, Bates DW. The incidence and severity of adverse events affecting patients after discharge from the hospital. Ann Intern Med. 2003;138(3):161-7.

9. Khan KZ, Ramachandran S, Gaunt K, Pushkar P. The Objective Structured Clinical Examination (OSCE): AMEE Guide No. 81. Part I: an historical and theoretical perspective. Med Teach. 2013;35(9):e1437-46. doi:10.3109/0142159X.2013.818634.

10. Hess J, Whelan JS. Making health literacy real: adult literacy and medical students teach each other. J Med Libr Assoc. 2009;97(3):221-4. doi:10. 3163/1536-5050.97.3.012.

11. Roberts DM, Reid JR, Conner AL, Barrer S, Miller KH, Ziegler C. A Replicable Model of a Health Literacy Curriculum for a Third-Year Clerkship. Teach Learn Med. 2012;24(3):200-10. doi:10.1080/10401334. 2012.692261 .

12. Chugh A, Williams MV, Grigsby J, Coleman EA. Better transitions: improving comprehension of discharge instructions. Front Health Serv Manag. 2009;25(3):11-32.

13. Coleman CA, Appy S. Health literacy teaching in US medical schools, 2010. Fam Med. 2012;44(7):504-7.

14. Manning KD, Kripalani S. The use of standardized patients to teach lowliteracy communication skills. Am J Health Behav. 2007;31(Suppl 1):S105-10. doi:10.5555/ajhb.2007.31.supp.S105.

15. KS J, KL B, Manning S, Rask K, KJ J, Terry A. Development and Implementation of a Health Literacy Training Program for Medical Residents. Med Educ Online. 2006;13(11):1-8.

16. Price-Haywood EG, Roth KG, Shelby K, Cooper LA. Cancer risk communication with low health literacy patients: a continuing medical education program. J Gen Intern Med. 2010;25(Suppl 2):S126-9. doi:10. 1007/s11606-009-1211-6.

17. Kern DE, Thomas PA, Hughes MA, eds. Curriculum Development for Medical Education: A Six-Step Approach. Vol Second. Baltimore, MA: Johns Hopkins University Press. 2009.

18. National Patient Safety Foundation. Ask Me 3. http://www.npsf.org/? page=askme3. Accessed August 25, 2015.

19. Weiss, BD. Health Literacy and Patient Safety: Help Patients Understand. Manual for Clinicians. Vol 2nd ed. American Medical Association Foundation. 2007.

20. Wallace LS, Rogers ES, Roskos SE, Holiday DB, Weiss BD. Brief report: screening items to identify patients with limited health literacy skills. J Gen Intern Med. 2006;21(8):874-7. doi:10.1111/j.1525-1497.2006. 00532.x.

21. Chew LD, Bradley KA, Boyko EJ. Brief questions to identify patients with inadequate health literacy. Fam Med. 2004;36(8):588-94.

22. Schillinger D, Piette J, Grumbach K, et al. Closing the loop: physician communication with diabetic patients who have low health literacy. Arch Intern Med. 2003;163(1):83-90.

23. Kountz DS. Strategies for improving low health literacy. Postgrad Med. 2009;121(5):171-7. doi:10.3810/pgm.2009.09.2065.

24. Agency for Healthcare Research and Quality (AHRQ). Care Transitions from Hospital to Home: IDEAL Discharge Planning Implementation Handbook. 2013.

25. R Gunning. The Technique of Clear Writing. McGraw-Hill. 1952.

26. McLaughlin GH. SMOG Grading — a New Readability Formula. J Read. 1969;12(8):639-46.

27. Flesch R. A new readability yardstick. J Appl Psychol. 1948;32(3):221-33. doi:10.1037/h0057532.

28. Stossel LM, Segar N, Gliatto P, Fallar R, Karani R. Readability of patient education materials available at the point of care. J Gen Intern Med. 2012;27(9):1165-70. doi:10.1007/s11606-012-2046-0.

29. Harper W, Cook S, Makoul G. Teaching medical students about health literacy: 2 Chicago initiatives. Am J Health Behav. 2007;31(Suppl 1):S11114. doi:10.5555/ajhb.2007.31.supp.S111.
30. Core Entrustable Professional Activities for Entering Residency: Curriculum Developers Guide. Vol American Association of Medical Colleges. 2014.

\section{APPENDIX 1: OSCE SCENARIO}

Back-of-door instructions for student:

Ms. Clotter is a 60-year-old woman, status post left hip replacement 1 month prior to admission, who presented 1 week ago with palpitations and hemoptysis, and was found to have a large pulmonary embolus. You have already bridged her from heparin to warfarin (Coumadin). Today she will be discharged home, and you must explain the discharge plan. Do not take a full history or perform a physical exam.

Please make sure that before discharging Ms. Clotter, you:

1. Assess her health literacy.

Ensure that she:

2. Understands her diagnosis of pulmonary embolus.

3. Will take $5 \mathrm{mg} \mathrm{PO}$ warfarin on $\mathrm{M} / \mathrm{W} / \mathrm{F}$ for at least 3 months.

4. Understands benefits and possible risks of warfarin.

5. Will go to weekly "Coumadin Clinic" on Thursdays to check her INR (first clinic is this Thursday).

\section{APPENDIX 2}

Table 3 Health Literacy Workshop and OSCE Student Survey

1. What month did you take Introduction to Internship?

2. Did you attend the health literacy workshop before the health literacy OSCE?

3. I will use the skills I learned in the workshop in clinical practice.

4. The health literacy workshop prepared me well for the health literacy OSCE.

5. The OSCE reinforced the skills taught in the workshop.

6. There was enough time to complete the required tasks of the OSCE.

7. After the workshop and OSCE, I feel confident that I can assess patients' health literacy.

8. After the workshop and OSCE, I feel confident that I can effectively communicate with patients with low health literacy.

9. Health literacy is important to consider in patient care.

10. Please list what specialty you will going into below. 